\title{
The Importance of Early Decompression Combined with Bone Grafting and Internal Fixation and Early Electric Stimulation in the Treatment of Severe Injury of Spinal Cord
}

\author{
Tang $\mathbf{C}^{1 *}$, Zhang $X^{1}$, Zhou $X^{1}$, Zhang $\mathbf{P}^{1}$, Zhao $\mathbf{S}^{1}$, Zhang J1, Ji Z1, Zhu D1, Cao X² and Cai W² \\ ${ }^{1}$ Department of Orthopedics of Sihong County People's Hospital of Jiangsu Province, Jiangsu Sihong, PR China \\ ${ }^{2}$ Department of Orthopedics of People's Hospital of Jiangsu province, Jiangsu Sihong, PR China
}

\begin{abstract}
Objective: To explore the anterior/posterior decompression, bone graft, internal fixation combined with postoperative early stimulation in the treatment of cervical spine trauma complicated with spinal cord injury treatment.
\end{abstract}

Methods: From 2005 January to 2014 December, 40 patients with cervical spine fracture, dislocation of injury associated with spinal cord injury were treated with anterior/posterior decompression, bone graft, internal fixation operation combined early electrical stimulation after operation.

Results: 40 cases were followed up from 12 months to 6 years. Bone graft healed well, no case of plate and screws loosening, rupture and other complications, symptoms of spinal cord injury improved satisfactorily. Preoperative spinal cord injury Frankel grade: A grade 24 cases, B grade 4 cases, C grade 5 cases, D grade 7 cases, postoperative recovery to $B$ grade 2 cases, E grade 26 cases. According to the Japanese Department of orthopedics Society (JOA) criteria score, postoperative improvement rate was $89.26 \%$.

Conclusion: Anterior/posterior spinal canal decompression, bone graft, early internal fixation operation combined with postoperative early electrical stimulation in the treatment of patients with cervical spine injury associated with spinal cord injury, can prevent or alleviate the secondary spinal cord injury and other complications, provide effective fixation spinal segmental stability, promote the recovery of spinal cord function, achieve a satisfactory effect.

Keywords: Cervical injury; Internal fixation; Bone grafting; Electrical stimulation; Spinal cord injury

\section{Introduction}

With the rapid development of building, transportation, cervical vertebra trauma cases gradually increased, most of them associated with spinal cord injury. Good effect has incomplete spinal cord injury in timely operation combined with hormone, nerve nutrition drugs after application, if not combined injury and other reasons timely decompression operation is easy to develop into a complete injury, and difficult to recover and disability. Spinal cord injury is relatively complex; spinal cord function recovery of poor curative effect, treatment of this condition can lead to different outcomes. In our hospital from January 2005 to December 2014, 40 cases of cervical spine fracture, dislocation and the original traumatic cervical disc degeneration in patients with cervical spinal cord injury based. The early anterior and posterior decompression, and internal fixation, with preoperative, hormone, dehydration agent application and electric stimulation therapy, significantly improved the treatment of spinal cord injury effect.

\section{Materials and Methods}

\section{Patient data}

From January 2005 to December 2014, 40 cases of cervical spinal cord injury were treated with the early decompression and internal fixation and electric stimulation therapy. Among of them, male 34 cases, female 6 cases. The age ranged from 22 to 71 years old, average 44.1 years old, including 1 cases of anterior dislocation of Atlas, the C3 vertebral fracture in 2 cases, the $\mathrm{C} 4$ vertebral fracture in 4 cases, the $\mathrm{C} 5$ vertebral fractures in 16 cases, the C6 vertebral fracture in 11 cases, 4 cases of the $\mathrm{C} 6$ vertebral dislocation, the $\mathrm{C} 67$ vertebral double fracture with the C6 vertebral dislocation in 2 case, merge adjacent segment degeneration of the intervertebral disc in 18 cases. The incomplete paraplegia in 16 cases, the paralysis in 24 cases. Preoperative spinal cord injury Frankel grade: A grade 24 cases, B grade 4 cases, C grade 5 cases, D grade 7 cases. Fusion segments: Single segment in 25 cases, 9 cases of double segment, 6 cases of multiple segmental. Operation approach: 32 cases of anterior approach; 3 case of posterior approach, 5 cases of anterior and posterior approach. Fixed position: the highest for the occipital bone, the lowest is T1. Operation time: $4 \mathrm{hrs}$ after injury to 5 days of operation, the average 6 hrs.

\section{Internal fixation}

Cervical anterior titanium plate, titanium mesh, posterior screw rod system or lateral mass plate (Shandong Weigao Department of orthopedics materials Limited) and Vertex internal fixation system (Sofamor Danek company).

\section{Operation method}

Simple anterior operation with the patient in the supine position, take the neck in neutral position, nasal or oral intubation, conventional

*Corresponding author: Tang C, Department of orthopedics of Sihong County People's Hospital of Jiangsu Province Jiangsu Sihong, PR China, Fax: +86-052786221474; E-mail: tangch168@sohu.com

Received November 17, 2014; Accepted March 28, 2015; Published March 31 2015

Citation: Tang C, Zhang X, Zhou X, Zhang P, Zhao S, et al. (2015) The Importance of Early Decompression Combined with Bone Grafting and Internal Fixation and Early Electric Stimulation in the Treatment of Severe Injury of Spinal Cord. J Trauma Treat 4: 239. doi:10.4172/2167-1222.1000239

Copyright: (C) 2015 Tang C, et al. This is an open-access article distributed under the terms of the Creative Commons Attribution License, which permits unrestricted use, distribution, and reproduction in any medium, provided the original author and source are credited. 
Citation: Tang C, Zhang X, Zhou X, Zhang P, Zhao S, et al. (2015) The Importance of Early Decompression Combined with Bone Grafting and Internal Fixation and Early Electric Stimulation in the Treatment of Severe Injury of Spinal Cord. J Trauma Treat 4: 239. doi:10.4172/21671222.1000239

sterile towel, on the right cervical do $6 \sim 8 \mathrm{~cm}$ long transverse incision, routine anterior cervical approach by exposing to prominent vertebra clearance, vertebral fracture and its operation within the scope of the adjacent last, the vertebrae. Fracture of vertebral resection of interverbebral disc, for vertebral, adjacent degenerative disc disease corpectomy for, vertebral, the operation area of intervertebral disc tissue, upper and lower bounds of distal vertebral body near, after thoroughly, on the edge of creeping decompression, posterior longitudinal ligament, removal, and then remove it, vertebral endplate and the front portion projecting osteophytes. Rinse cavity treatment, gelatin sponge implantation corpectomy rear, traction backspin is cervical vertebra, the vacuum obtaining vertebral cancellous bone into the titanium mesh and autogenous iliac bone defect after finishing the traction in after decompression (titanium mesh to cross 2 vertebral body, and keep on the side and vertebral endplate, and insert locking). In order to ensure the fixation position is correct, choose the appropriate length of steel plate is placed in the anterior vertebral (proximal upper screw in the distal screw, vertebra in the middle part of the vertebral body), followed by line drilling, probe sounding, before plate recently, distal diagonal screw 2 cancellous screws, then turn into another diagonal pine bone screws, screw tail anti exit bolt respectively rotate 180 degrees to prevent screw back. Complete steel plate and internal fixation, bone to bone block stability to decide whether to fix the screw fixation and bone graft blocks. Postoperative C-arm X-ray perspective that screws are not entering the spinal canal. Posterior operation, prone position, the median incision decompression, reduction, fixation. In this group, 1 cases of absence of the posterior arch of atlas with atlantoaxial dislocation, was fixed with Vertex internal fixation system for the occipital bone, $\mathrm{C} 2,3$, pedicle or lateral mass fixation, graft bone fusion after reduction. 2 cases combined with posterior pedicle fracture was fixed with posterior cervical fixation, graft bone fusion after decompression. Combined anterior and posterior approach, before the prone position, posterior midline incision of vertebral injury after routine exposure of lower and upper cervical lateral mass, distraction, poking reduction correct locked facet, the Shandong Weigao Department of orthopedics materials limited posterior screw rod fixation system or the lateral mass plate fixation of cervical lateral mass, spinal canal decompression, side a lateral bone. Closure mouth after anterior operation, with the former method. Anterior and posterior fixation, fusion segments corresponding to the best. In 40 cases of this group, operation time was $130 \sim 480 \mathrm{~min}$, average $216 \mathrm{~min}$; bleeding was $80 \sim 800 \mathrm{ml}$, average $160 \mathrm{ml}$.

\section{Postoperative treatment}

After operation, in addition to conventional hormone application, dehydrating agent, nerve nutrition drugs improving microcirculation (methylprednisolone, gangliosides, 654-2, methylcobalamin) application processing, the other using electrical stimulation (Beijing baorunjia Technology Co., Ltd. CRI-A electric brain rehabilitation instrument), the application of the prevent disuse muscle atrophy, muscle paralysis, the neuromuscular rehabilitation, is promoting nerve muscle of the three prescription turns or alternately, strength differ from man to man (30 70 mA), mainly in the patients feel, combined with the patient body reaction condition. The treatment time per day for 1 to 2 hours. Anterior operation in patients with neck support protection of 4 6 weeks after removal, multi-segment fusion of head and neck thoracic brace for 3 months. On postoperative day and 4,8 weeks and 3 months. X-ray film, in order to understand the internal fixation system position and bone healing. Also can be examined by MRI, to understand whether thorough decompression and internal changes in the spinal cord.

\section{Results}

All the 40 cases were followed up from 12 months to 6 years, average 30 months. Bone graft healed well, no case of plate and screw loosening, fracture and other complications, symptoms improved with paraplegia. Patients with dislocation of cervical vertebra anterior titanium plate, titanium mesh and bone graft in January after no nursing bed self-care, not combined with rehabilitation exercise, postoperative cervical sequence changes occurred in March, two times of operation of posterior lateral mass plate fixation, and bone grafting fusion, March photograph tips satisfaction. In 40 patients after spinal cord injury recovered to Frankel grade B 2 cases, E grade 38 cases. According to Japanese Society (JOA) Department of orthopedics preoperative evaluation standard score, 12 months postoperative improvement rate was $89.26 \%$ (Table 1 and Figures 1-4).

\section{Discussion}

\section{Operation time of cervical spine trauma, fracture and dislocation with spinal cord injury}

Injury of cervical spine fracture, dislocation, treatment of patients with spinal cord injury has been in a process of continuous exploration, development, treatment effect is not ideal, the disability rate is up to $67 \%$ [1]. Spinal cord injury is potentially reversible injuries, the recovery of spinal cord function associated with spinal cord compression time inverse, early decompression can promote the recovery of nerve function; reduce the scope of nerve damage. Experimental drugs, treatment is aimed at relieving the secondary injury of spinal cord [25], Inhibiting the inflammatory reaction, reduce edema in spinal cord injury play a very important role in comprehensive treatment [6], the relatively lower spinal buffer space requirements.

\begin{tabular}{|c|c|c|c|c|c|}
\hline Injury segment of cases & $\begin{array}{c}\text { The } \\
\text { number of cases }\end{array}$ & $\begin{array}{c}\text { Preoperative JOA score } \\
\text { accumulated }\end{array}$ & $\begin{array}{l}1 \text { Month postoperative } \\
\text { JOA score accumulated }\end{array}$ & $\begin{array}{l}3 \text { Months postoperative } \\
\text { JOA score accumulated }\end{array}$ & $\begin{array}{l}12 \text { Months postoperative } \\
\text { JOA score accumulated }\end{array}$ \\
\hline $\mathrm{C} 1$ & 1 & 8 & 17 & 17 & 17 \\
\hline $\mathrm{C} 3$ & 3 & 2 & 51 & 51 & 51 \\
\hline $\mathrm{C} 4$ & 3 & 3 & 51 & 51 & 51 \\
\hline C5 & 16 & 8 & 272 & 272 & 272 \\
\hline C6 & 7 & 0 & 119 & 119 & 119 \\
\hline $\mathrm{C} 4-5$ & 3 & 0 & 16 & 16 & 16 \\
\hline C4-6 & 2 & 0 & 9 & 10 & 10 \\
\hline C5-6 & 2 & 0 & 1 & 1 & 1 \\
\hline C5-7 & 2 & 0 & 1 & 1 & 1 \\
\hline
\end{tabular}

Preoperative, postoperative JOA score were significant difference, $\mathrm{P}<0.001$

Table 1: Comparison of preoperative, postoperative JOA score of the patients. 
Citation: Tang C, Zhang X, Zhou X, Zhang P, Zhao S, et al. (2015) The Importance of Early Decompression Combined with Bone Grafting and Internal Fixation and Early Electric Stimulation in the Treatment of Severe Injury of Spinal Cord. J Trauma Treat 4: 239. doi:10.4172/21671222.1000239

Page 3 of 4

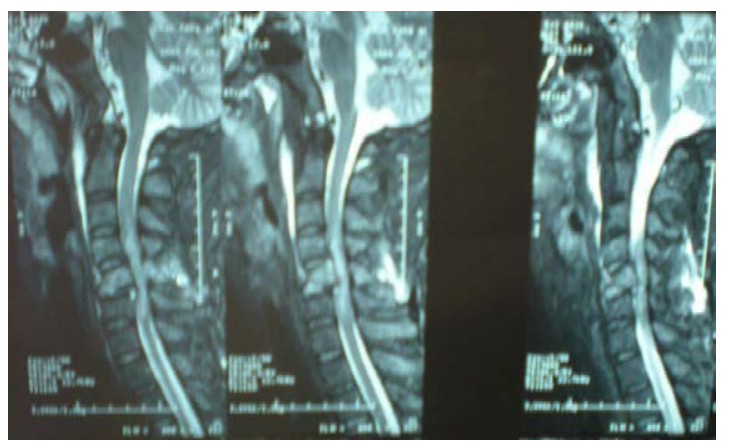

Figure 1: C5 vertebral fracture with posterior vertebral olisthy, complete paralysis of preoperative MRI film showed spinal cord contusion, bleeding, swelling, buffer space of intraspinal disappear.

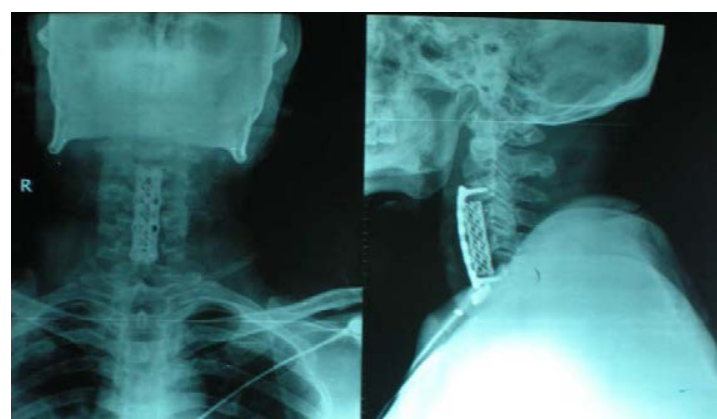

Figure 2: C5 vertebral fracture with posterior vertebral olisthy, complete paralysis of anterior 2 corpectomy, decompression, titanium mesh cage bone $C R$ reexamination after internal fixation of paraplegia recovered satisfactorily.

Although the cervical vertebra trauma fracture, dislocation with spinal cord injury, nerve root compression time for operation patients within 24 hours of operation is better, but the severe spinal cord injury patients with spinal buffer space is very small, in the conditions with methylprednisolone impact at the same time, except for the presence of severe compound injury unconditional early operation, operation the sooner the better [7]. Clinically, especially should prevent damage secondary spinal cord edema of spinal cord blood for the impact, to avoid the occurrence of spinal cord compression syndrome (similar osteofascial compartment syndrome) induced secondary spinal cord ischemia caused irreversible damage, seriously affect the treatment effect. Our experience of cervical spinal cord contusion in severe cases, compression, processing the link from the wounded site, hospital inspection shall keep the neck brake, avoid the two injury. Pathological and clinical study showed that operation as soon as possible and strive in 6 8 hours for good (at all levels of hospitals should be as short as possible inspection, consultation, preoperative preparation process, emergency MRI examination, good anesthesia risk assessment), to prevent irreversible injury secondary to spinal cord ischemia, degeneration. Many patients with multiple trauma conditions do not permit and other reasons cannot be timely operation cause postoperative lifelong paralysis ( 2 cases in this group of poor recovery). Such as spinal cord contusion, compression is not serious with compound injury unstable vital signs, the correct shock, neck brake, dehydrating agent, application of hormone treatment can ensure the blood supply of spinal cord, operation timing of decompression can be appropriately delayed ( 2 cases in this group of patients with complete spinal cord injury after injury, $4 \sim 5$ days without recovery after operation, also recovered rapidly and achieved satisfactory curative effect).
Although some complete spinal cord injury may have spinal cord concussion, shock factor, even if the imaging data showed spinal cord compression is not serious, but if the waiting more than 24 hours decompression may be permanent loss due to spinal cord contusion, edema and secondary spinal cord ischemia caused by spinal cord damage, to improve the quality of life of patients, reduce the burden of care, a timely MRI examination confirmed spinal cord transection of the cases are still not as far as possible emergency operation treatment. The diagnosis of spinal cord transection of the case has been, due to the current stem cell transplantation still failed to reach the ideal effect, can choose appropriate operation timing of treatment, to ensure the safety of patients. This group of patients with postoperative improvement rate higher than reported, consider the early operation plays a key role.

\section{Operation approach, decompression method}

Anterior, posterior or anterior posterior decompression, bone grafting and internal fixation operation has been widely used in cervical surgery $[8,9]$, the fixation of various types, some easy to produce poor fusion, cervical instability and other complications, fixed materials gradually be eliminated, and the anterior cervical locking titanium plate, screw rod with titanium mesh the system application can meet various types of internal fixation for the treatment of cervical spine injury, has stable curative effect, has become the preferred materials for internal fixation of unstable cervical spine injury. Should be based on the specific type of injury, spinal cord compression and location of the injury situation determines the operation approach, and fully consider the secondary spinal cord edema may bring influence the blood supply of spinal cord. Because of spinal cord injury caused by cervical trauma for many of the intervertebral disc, spinal cord compression fractures from the front and nerve root decompression, the most effective. A few cases are locked facet, or rear fragments, ligamentum flavum hypertrophy of spinal cord compression of the spinal cord, even before there is oppression, posterior or anterior or road before and after posterior decompression and fixation, the corresponding segment, bone graft fusion and stabilization of the spine, ensure the operation effect. Part of the cervical fracture dislocation with spinal cord injury, combined with the adjacent segment degeneration of cervical intervertebral disc, the effects on the blood supply of spinal cord segments should handle. And the longitudinal ligament ossification, hypertrophy, to exist after resection of posterior longitudinal ligament, achieve complete decompression. Patients with spinal decompression fully estimate the buffer space, on the front, back and both before and after spinal cord compression caused by structure clear, we should judge after spinal cord injury secondary to edema can obtain the buffer space to ensure the blood supply of spinal cord anterior, posterior, respectively or combined anterior and posterior approach operation. Combined anterior and posterior operation suggestion has road, anterior posterior anterior operation, preventing oozing of blood of respiration. In the spinal posterior longitudinal ligament flavum trailing edge to the corresponding edge distance is less than the spinal anteroposterior diameter case, operation should be implemented as soon as possible, don't let slip the opportunity of. On the existence of chipping disc protruding into the spinal canal or adjacent segment degeneration of the intervertebral disc, the subtotal resection of vertebral body (stenosis of the spinal canal and spinal cord edema is not serious can carefully select the part retained the vertebral bone, prevent the titanium mesh after the shift), avoid intervertebral approach difficult operation, spinal cord injury, and fully expand the buffer space within the spinal canal. With case column injury instability, as far as possible combined anterior posterior approach decompression, internal fixation. 
Citation: Tang C, Zhang X, Zhou X, Zhang P, Zhao S, et al. (2015) The Importance of Early Decompression Combined with Bone Grafting and Internal Fixation and Early Electric Stimulation in the Treatment of Severe Injury of Spinal Cord. J Trauma Treat 4: 239. doi:10.4172/21671222.1000239
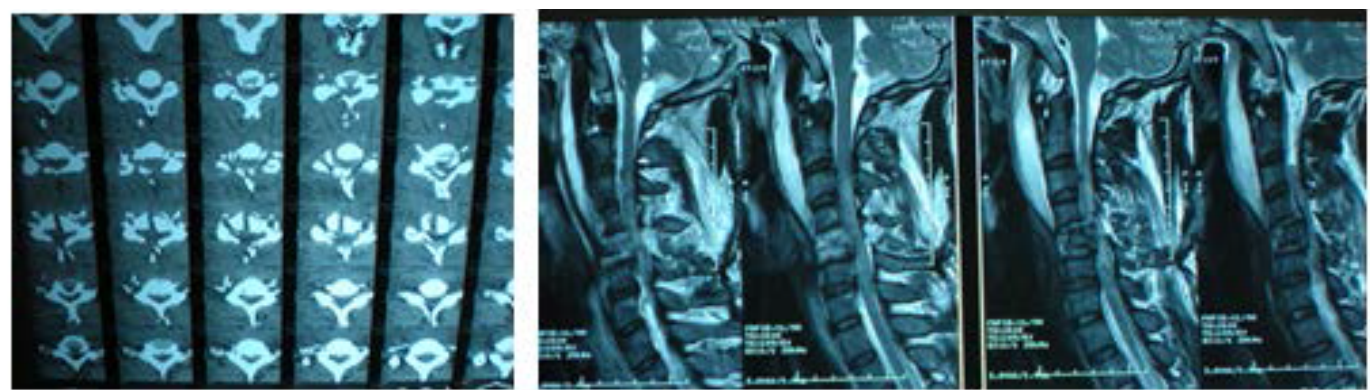

Figure 3: C4 spondylolisthesis, C5 column fracture with complete paralysis of preoperative CT, MRI films showed spinal cord contusion and hemorrhage, edema, spinal stenosis.

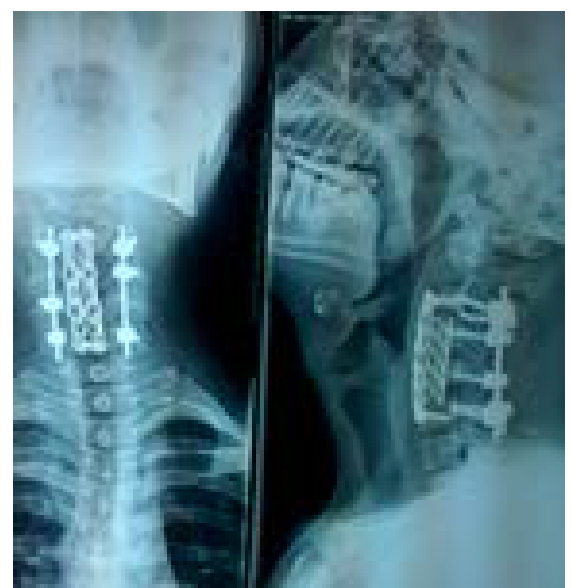

Figure 4: C4 spondylolisthesis, C5 anterior and posterior column fracture with complete paralysis after expanding decompression, internal fixation after CR reexamination of paraplegia recovered.

\section{Electrical stimulation treatment}

Electrical stimulation can be through a variety of mechanisms for the recovery of the spinal cord functions play a good role in promoting [10], the patients with positive after operation, in addition to the nerve nutrition, improve microcirculation, dehydration and other drug treatment, the full application of electrical stimulation and rehabilitation equipment, comprehensive treatment, can obviously promote the spinal cord, nerve function recovery, satisfactory curative effect. 10 cases of this group with complete spinal cord injury function recovery, the daily electrical stimulation treatment for 1 to 2 hours, recovered satisfactorily. After 3 months can walk independently, part of patients discharged from hospital after a hyperalgesia phenomenon, the active request after the treatment of electric stimulation on rapid remission or recovery, reduce the rate of disability. Combined with electrical stimulation treatment on the recovery of spinal cord function is not obvious, can consider to hyperbaric oxygen therapy.

Previous and current study and their limitations and directions for further research of cervical spine trauma, fracture and dislocation with spinal cord injury

Previous studies have focused on the mechanism of spinal cord injury and research on drug treatment. But the replication model of spinal cord injury usually cannot be a true reflection of the injured degree of spinal cord. Study on spinal cord blood supply has not been enough attention. Current research suggests that while in the active drug in the treatment of spinal cord injury, the effect of early operation of decompression, hyperbaric oxygen therapy is good. But the existence of some deficiencies of large dose methylprednisolone used side effects, patients with on-site treatment ineffective, to the lack of specialist hospital treatment time, the hospital inspection booking time is long, the emergency operation physicians less, operation timing of decompression indication is not clear (such as finger replantation), with spinal cord injury treatment center received hyperbaric oxygen treatment lack of timely, and so on.

In the future, to establish model of spinal cord injury, should pay attention to uniformity force of spinal cord injury, integrity damage of the spinal canal, the timing of early spinal bony decompression, further study at the same time for spinal cord decompression indications, timing. The establishment of spinal cord injury treatment center more, drugs inhibiting the inflammatory reaction developed with smaller side effect, prolonging the spinal cord injury of local mild hypothermia cooling time. For those cases of spinal cord transection, further study of transplantation of neural stem cells and spinal shortening of spinal cord after anastomosis or for future research direction.

\section{References}

1. Liao W, Zhang G (2003) To further strengthen the research on repair of spinal cord injury. Chinese Journal of spine and spinal cord 13: 517-519.

2. Tang C, Cao X, Wang D (2002) Effects of nerve growth factor on spinal neuronal apoptosis after spinal cord injury. Journal of Nanjing Medical University 22 203-207.

3. Tang C, Cao X, Wang D (2002) Effects of nerve growth factor on neuronal nitric oxide production after spinal cord injury in rats. Chin J Traumatol 5: 165-168.

4. Tang C, Luo Y, Cao X (2002) Effects of nerve growth factor on neurons of $\mathrm{N}$ methyl -D- aspartate receptor and nitric oxide after spinal cord injury. Chinese Journal of experimental surgery 19: 179 .

5. Tang C, Cao X, Wang D (2002) Effects of Nerve Growth Factor on Bcl-2 Protein after Spinal Cord Injury in Rats. Journal of Nanjing Medical University 16: 112-114.

6. Xue H, Zhang XY, Liu JM, Song Y, Liu TT, et al. (2013) NDGA reduces secondary damage after spinal cord injury in rats via anti-inflammatory effects. Brain Res 1516: 83-92.

7. Jazayeri SB, Firouzi M, Abdollah ZS, Saeedi N, Pirouz E, et al. (2013) The effect of timing of decompression on neurologic recovery and histopathologic findings after spinal cord compression in a rat model. Acta Med Iran 51: 431437.

8. Heidecke V, Rainov NG, Burkert W (1998) Anterior cervical fusion with the Orion locking plate system. Spine 23: 1796-1803.

9. Rehman L, Mohammad HAS, Qayoom KHA, Arain SH, Ali S, et al. (2013) Anterior decompression and fixation with Webb-Morley procedure in dorsolumbar spinal injury. J Coll Physicians Surg Pak 23: 330-333.

10. Wu Y, Collier L, Qin W, Creasey G, Bauman WA, et al. (2013) Electrica stimulation modulates Wnt signaling and regulates genes for the motor endplate and calcium binding in muscle of rats with spinal cord transection. BMC Neurosci 14: 81. 